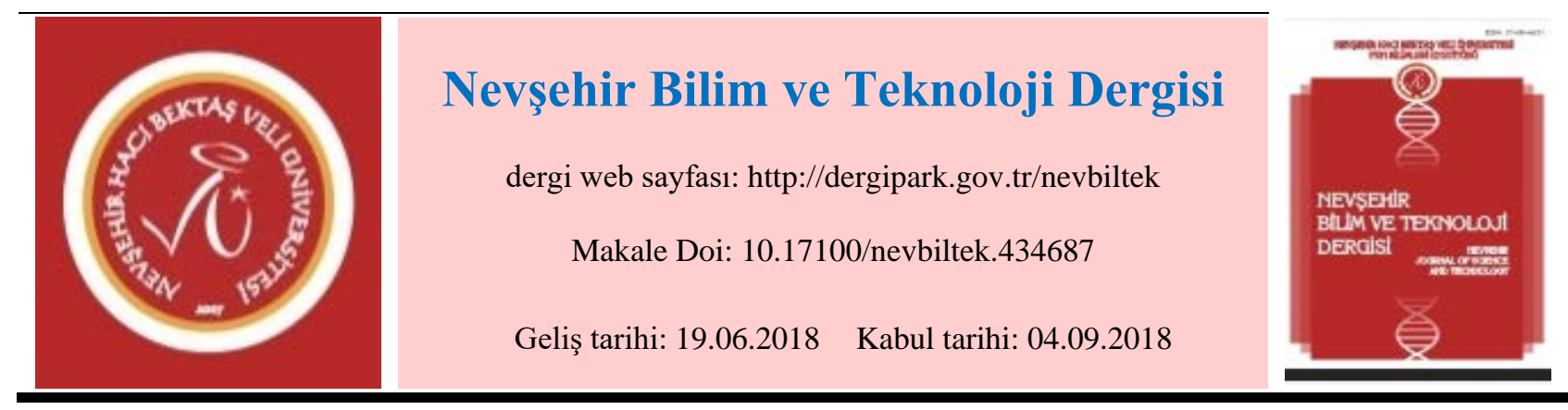

\title{
Metal Katkılı MOF-5 İçeren Karışık Matriksli Membranlar İle Hidrojen Geçirgenliğinin İyileştirilmesi $^{1}$
}

\author{
Hülya AYKAÇ ÖZEN ${ }^{1,}$, Bahtiyar ÖZTÜRK ${ }^{2}$ \\ ${ }^{1}$ Ondokuz Mayıs Üniversitesi, Mühendislik Fakültesi, Çevre Mühendisliği Bölümü, Samsun \\ ORCID ID: 0000-0003-4990-6682 \\ ${ }^{2}$ Ondokuz Mayls Üniversitesi, Mühendislik Fakültesi, Çevre Mühendisliği Bölümü, Samsun \\ ORCID ID:0000-0002-3385-0701
}

Öz

\begin{abstract}
Hidrojen, yüksek enerji yoğunluğu ve çevreye zarar vermeyen özellikleri ile geleceğin enerji taşıyıcısı olarak görülmektedir. Doğada saf halde bulunmayan hidrojen, gaz karışımından çeşitli tekniklerle ayrılabilmektedir. Bu ayırma prosesleri içerisinde yer alan membran teknolojisi, enerji verimliliğ̣i ve mekanik dayanıklılı̆g sayesinde son yıllarda dikkati üzerine çekmiştir. Membran teknolojileri içinde yer alan ve membran içerisine dolgu maddesi ilavesiyle oluşturulan karışı matriksli membranlar (MMM) ile gaz ayırma özelliklerinin iyileştirildiği görülmüştür. Karışık matriksli membranlarda dolgu maddesi olarak kullanılan MOF yapıları da yüksek yüzey alanları ve gözenek hacimlerinden dolayı tercih edilmekte ve gaz geçirgenliğine olumlu etki yapmaktadır. Bu çalışmada, iki farklı metal kullanarak NiCoMOF-5 yapısı sentezlenmiştir. Karışık matriksli membran hazırlamak üzere poliimit membrana NiCoMOF-5 ilave edilmiştir. Oluşturulan yapılar çeşitli karakterizasyon işlemlerine tabi tutulmuştur. Sonuçlar, karışık matriksli membranın başarılı bir şekilde sentezlendiğini ve MOF ilave edilen poliimit membranın saf poliimide göre gaz geçirgenlik performansını arttırdığını göstermiş̧ir.
\end{abstract}

Anahtar kelimeler: Hidrojen, Metal Organik Kafes, Karışık Matriksli Membran

\section{Improvement of Hydrogen Permeability with Mixed Matrix Membranes Containing Metal Doped MOF-5}

Abstract

Hydrogen is seen as the energy carrier of the future with its high energy density and envrironmental friendly properties. Since hydrogen is not pure in nature, it can be separated from the gas mixture by various techniques. Membrane technology involved in hydrogen separation processes has attracted attention in recent years thanks to its energy efficiency and mechanical strength. It has been found that the mixed matrix membranes formed by the addition of the filler material inside the membrane technology improve the gas separation properties. MOF structures used as a filler material are also preferred due to their high surface area and pore volume and have a positive effect on gas permeability. In this work, NiCoMOF-5 structure was synthesized using two different metals. Then, NiCoMOF-5 was added into polyimide membrane to prepare a mixed matrix membrane. The obtained MOF and MMM structures were evaluated various characterization procedures. The results show that the mixed matrix membrane is successfully synthesized and that the MOF added polyimide membrane improves the gas permeability performance relative to the pure polyimide.

Keywords: Hydrogen, Metal Organic Framework, Mixed Matrix Membrane

\footnotetext{
${ }^{11} \mathrm{Bu}$ makalenin tamamı 10-12 Nisan 2018'de Çeşme'de gerçekleștirilen "The Internatinonal Conference on Materials Science Mechanical and Automotive Engineerings and Technology (IMSMATEC’18)" konferansında sunulmuştur.

Sorumlu yazar e-mail: hulya.aykac@omu.edu.tr
} 


\section{Giriş}

Hidrojen; doğada bol miktarda bulunması, enerji içeriğinin diğer yakıtlara göre yüksek olması, elektrik enerjisine kolaylıkla dönüşebilmesi, son ürün olarak sadece ortama su vererek çevre dostu olması ile geleceğin enerji stratejileri arasında önemli bir yer almaktadır [1-4]. Üretim aşamasında saf olarak elde edilemeyen hidrojenin bulunduğu gaz karışımından ayrılması gerekmektedir. Hidrojenin basınç salınımlı adsorpsiyon ve kriyojenik distilasyon gibi yöntemlerle gaz karışımından ayrılması mümkün olsa da, son yıllarda membran ile gaz karışımından ayrılması göze çarpan bir yöntem olarak literatürde yer almaktadır. Çünkü, membranların işletimi kolay, yatırım maliyeti düşük ve enerji tüketimi diğer yöntemlere göre daha azdır [5]. Membranlar, gaz ayırımında polimerik, inorganik ve karışık matriksli membranlar olarak sınıflandırılmaktadır. Polimerik membranlarda, geçirgenlik ve seçicilik arasında ters orantı olduğundan hem geçirgenlik hemde seçiciliklerini aynı anda arttırmak mümkün değildir. İnorganik membranların ise üretiminin pahalı olması, sınırlı sayıda yapı oluşturması ve oldukça kırılgan olması kullanımını zorlaştırmaktadır [6]. Karışık matriksli membranlar (MMM) polimerik ve inorganik membranlara alternatif olarak ortaya çıkmış, polimerik membrana dolgu maddesinin ilavesiyle oluşturulmuş membranlardır [7]. Dolgu maddeleri arasında, metal organik kafes sistemleri (MOF) diğer dolgu maddelerine göre üstün özellikleri ile ilgi çekmiştir. MOF’lar, yüksek yüzey alanları, hızlı ve kolay bir şekilde sentezlenebilmeleri, gözenek boyutu ve şekillleri ayarlanarak sonsuz miktarda yapı oluşturabilmeleri ile hidrojen ayrımında potansiyel olarak görülmektedir [8]. Metal organik kafes grubu ailesinden olan MOF-5, benzendikarboksilik asit ve $\left[\mathrm{Zn}_{4} \mathrm{O}\right]^{6+}$ kümeleriyle oluşmuş [9] ve kontrol edilebilen poroziteleri ile dolgu maddesi olarak kullanılmaktadır. Günümüzde MOF-5 ile hazırlanan karışık matriksli membranlar ile birçok çalışma yapılmış ve MMM'ların gaz ayırma performansları arttırılmıştır. Perez ve arkadaşları (2009), Matrimid membrana MOF-5 ilave ederek oluşturdukları karışık matriksli membranda gaz geçirgenliklerini \%120 arttırmışlardır [7]. Başka bir çalışmada, MOF-5/PEI karışık matriksli membranda $\mathrm{H}_{2}$ geçirgenliği \%40 oranında artış göstermiştir [6]. Ancak MOF-5'in neme karşı hassas olması deney sonuçlarında farklılık olmasına neden olmuş ve son yıllarda yapılan çalışmalar MOF-5 yapısına metal ilave edilmesi bu sorunun çözüldüğü ayrıca membranların performansını arttırdığını göstermiştir. Li ve arkadaşları (2012), MOF-5 yapısına Ni metali ilave ederek saf MOF-5’e göre daha yüksek yüzey alanı ve daha geniş gözenek elde edilmesini sağlamıştır [9]. Yine başka bir çalışmada, Co katkılı MOF-5’in oldukça kristal partiküllere ve yüksek yüzey alanına sahip olduğu raporlanmış ve gas adsorpsiyon özelliklerini iyileştirdiği görülmüştür [10].

$\mathrm{Bu}$ çalışmada, MOF-5 yapısına ilave edilen metallerin gaz geçirgenliğine etkisini anlamak için Kobalt (Co) ve Nikel (Ni) metalleri ile NiCoMOF-5 yapısı oluşturulmuştur ve bu yapı, karışık matriksli membran oluşturmak üzere poliimit membrana ilave edilmiştir. Hazırlanan MOF yapısı, taramalı elektron mikroskobu (SEM), fourier dönüşümlü kızılötesi spektroskopisi (FTIR) ve termogravimetrik analiz (TGA) ile karakterize edilmiştir. Üretilen karışık matriksli membranlara gaz geçirgenlik testi uygulanarak, bu yapıların gaz geçirgenlikleri irdelenmiştir.

\section{Materyal ve Metot}

Bu çalışmada, MOF sentezi ve karışık matriksli membran üretimi, MOF ve membranın karakterizasyonu ile geçirgenlik testleri gerçekleştirildi. Poliimid, Alfa Aesar firmasından satın alındı. Benzen-1,4-dikarboksilik asit(BDC, $>99 \%), \mathrm{Zn}\left(\mathrm{NO}_{3}\right)_{2} \cdot 6 \mathrm{H}_{2} \mathrm{O}, \mathrm{Ni}\left(\mathrm{NO}_{3}\right)_{2} \cdot 6 \mathrm{H}_{2} \mathrm{O}$ ve $\mathrm{Co}\left(\mathrm{NO}_{3}\right)_{2} \cdot 6 \mathrm{H}_{2} \mathrm{O}, \mathrm{N}, \mathrm{N}$-dimetilformamit $\left(\mathrm{DMF}, 99.8 \%, \mathrm{H}_{2} \mathrm{O}<\right.$ 0.15\%) ve N-metil-2-Pirolidon(NMP) Sigma-Aldrich firmasından temin edildi. Tüm kimyasallar ve çözücüler hiçbir ön işleme tutulmadı. Geçirgenlik testleri, \%99.99 saflıktan daha yüksek $\mathrm{H}_{2}$ gazı kullanılarak gerçekleştirildi.

\subsection{NiCoMOF-5 Sentezi}

NiCoMOF-5 yapısı daha önce yayınlanan prosedüre uygun olarak solvotermal yöntem ile hazırlandı [11]. 0,5 mmol çinko(II) nitrat hekzahidrat, 0,5 mmol kobalt(II) nitrat, 0,5 mmol nikel(II) nitrat hekzahidrat ve 0,5 mmol terefitalik asit bir cam şişeye konuldu ve üzerine $16 \mathrm{~mL}$ DMF ve $4 \mathrm{~mL}$ etanol ilave edilerek ultrasonik banyo yardımıyla çözündü. Numune şişesinin ağZı kapatılarak etüvde $110^{\circ} \mathrm{C}$ 'de 4 saat bekletildi. Etüvden çıkarılan şişe oda sıcaklığına 
getirilerek kristal yüzeyinde kalmış olabilecek DMF'in uzaklaştırılması için etanol ile yıkandıktan sonra vakum altında $60^{\circ} \mathrm{C}^{\prime}$ de 4 saat kurutuldu. Elde edilen partiküller ağzı kapalı bir şişede muhafaza edildi.

\subsection{Saf Poliimit Membran Üretimi}

Poliimit membran (PI) önceki çalışmalardan yararlanarak hazırlandı [12]. Yapılan ön çalışmalar neticesinde en uygun çözücü/polimer oranının 10/90 olarak belirlendi. Çözücünün (NMP) polimeri tamamen çözmesi için çözelti yaklaşık 12 saat boyunca $70^{\circ} \mathrm{C}$ 'de karıştırıcıda karıştııılarak homojen bir çözelti elde edildi. Döküm işlemi yapılmadan önce cam levhaların yüzeyi alkol ile temizlendi ve daha sonra belli dökme kalınlığında hazırlanmış baget kullanılarak (500 $\mu \mathrm{m}$ membran dökme kalınlığına sahip) polimerik çözelti cam levha üzerine döküldü. Cam levha $70^{\circ} \mathrm{C}$ ve 500 mmHg azot gazı atmosferinde vakum etüvüne yerleştirilerek membran yapısındaki çözücünün uçurulması için 12 saat bekletildi. Etüvden çıkarılan cam levha oda sıcaklığına getirildikten sonra su içine daldırılarak cam üzerinden membranın ayrılması sağlandı. Cam plakadan ayrılan membran tavlanmak üzere daha yüksek sıcaklıkta $\left(220^{\circ} \mathrm{C}\right)$ etüve yerleştirildi ve yaklaşık 24 saat kadar vakum altında etüvde tavlanan membran uygun çapta kesilerek gaz geçirgenlik performansı belirlenmek üzere gaz ayırma hücresine yerleştirildi. Membranın kalınlığı en az 5 noktasından mikrometre ile ölçülerek ( \pm 1 mikron hata ile) ortalama membran kalınlığı belirlendi.

\subsection{Karışık Matriksli Membran Üretimi}

NiCoMOF-5 içeren MMM hazırlamak için bir cam tüp içine $0,5 \mathrm{~g}$ poliimit, 4,5 g NMP ve diğer bir cam tüp içine $0,025 \mathrm{~g}$ NiCoMOF-5 ve 4,5g NMP koyuldu. Her iki cam tüp sicaklığ $70^{\circ} \mathrm{C}$ olan ultrasonik su banyosuna yerleştirildi ve 2,5 saat boyunca poliimidin çözünmesi ve MOF-5'in tamamen karışması sağlandı. Bu süre sonunda iki karışım birleştirilerek 5 saat boyunca sıcaklığ ${ }_{1} 70^{\circ} \mathrm{C}$ olan manyetik karıştırıcıda karıştırıldı. Yüzeyi temizlenmiş olan cam üzerine karışım dökülerek bagetle yayılması sağlandı. Daha sonra cam levha, çözücünün uçurulması amacı ile vakum etüvüne $70^{\circ} \mathrm{C}$ de $500 \mathrm{mmHg}$ Azot atmosferinde yerleştirildi. Yaklaşık 12 saat sonra cam plaka etüvden çıkarıldı ve membran cam yüzeyinden ayrıştırıldı. Cam plakadan ayrılan membran tavlanmak üzere vakumlu etüve yerleştirildi ve etüvün sıcaklığı kademeli olarak $220^{\circ} \mathrm{C}$ 'a çıkarıldı; membran bu sıcaklıkta 24 saat boyunca tavlandı. Tavlanan membrandan ayırma hücresine yerleştirilecek boyutta kesilerek önce 5 farklı noktadan kalınlığı ölçüldü ve daha sonra ayırma hücresine yerleştirilerek gaz geçirgenlik deneyleri gerçekleştirildi

\section{Bulgular}

\subsection{PI, NiCoMOF-5 ve MMM'm Karakterizasyonu}

Bu çalışmada, MOF-5 ve farklı metallerle modifiye edilen kafes yapılarının termal kararlılıklarını ve sıcaklığa bağlı olarak meydana gelen ağırlık kayıplarını belirleyebilmek için eş zamanlı TGA/DTA/DTG termik analiz teknikleri uygulanmıştır. Termik analiz eğrileri, $30^{\circ} \mathrm{C}$ ile $900^{\circ} \mathrm{C}$ arasında $40 \mathrm{~mL} / \mathrm{dk}$ inert $\mathrm{N}_{2}$ atmosferinde, $20^{\circ} \mathrm{C} / \mathrm{dk} 1 \mathrm{sitma} \mathrm{hızında}$ kaydedilmiştir.

Yapısında Zn'ye ilave olarak iki farklı metal içeren NiCoMOF-5 kristallerinin termik analiz eğrileri Şekil 3.1 'de verilmiştir. Üç aşamalı ağırlık kaybı sergileyen bu eğrinin, birinci aşamasındaki ağırlık kaybı $182-360^{\circ} \mathrm{C}$ arasında gerçekleşmiştir ve ağılık kaybı \%19'dur. Bu aşama, çözücü (DMF) ve adsorbe olmuş suyun uzaklaşmasına karş̧1ık gelmektedir. $408-557^{\circ} \mathrm{C}$ arasında meydana gelen ikinci ağırlık kaybı ise organik ligandların dekompozisyonuna karş1llk gelmektedir. $789^{\circ} \mathrm{C}$ 'den sonra inorganik oksitlerin (Ni/Co/Zn oksitler) oluştuğu düşünülmektedir. 
Nevşehir Bilim ve Teknoloji Dergisi (2018), 7(2) 166-173

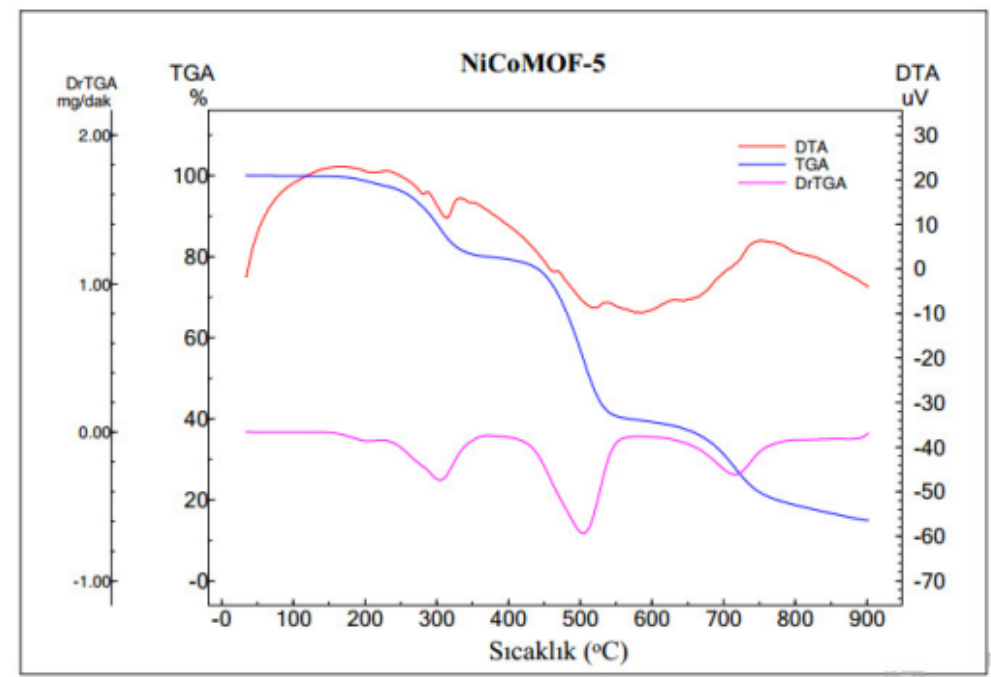

Şekil 3.1. NiCoMOF-5 yapısının TGA/DTA/DTG eğrileri

Saf poliimide metal katkılı MOF-5 yapısının ilave edilmesiyle oluşturulan karışık matrisli membranların termal kararlılığ Şekil 3.2'de görülmektedir. Saf poliimit ve NiCoMOF-5/PI membran birbirine oldukça benzer eğriler sergilemektedir. $\mathrm{Bu}$ sonuç poliimit yapısına metal katkılı MOF-5 eklendiğinde yapının termal kararlılı̆̆ının dikkate değer bir şekilde değişmediğini göstermektedir.

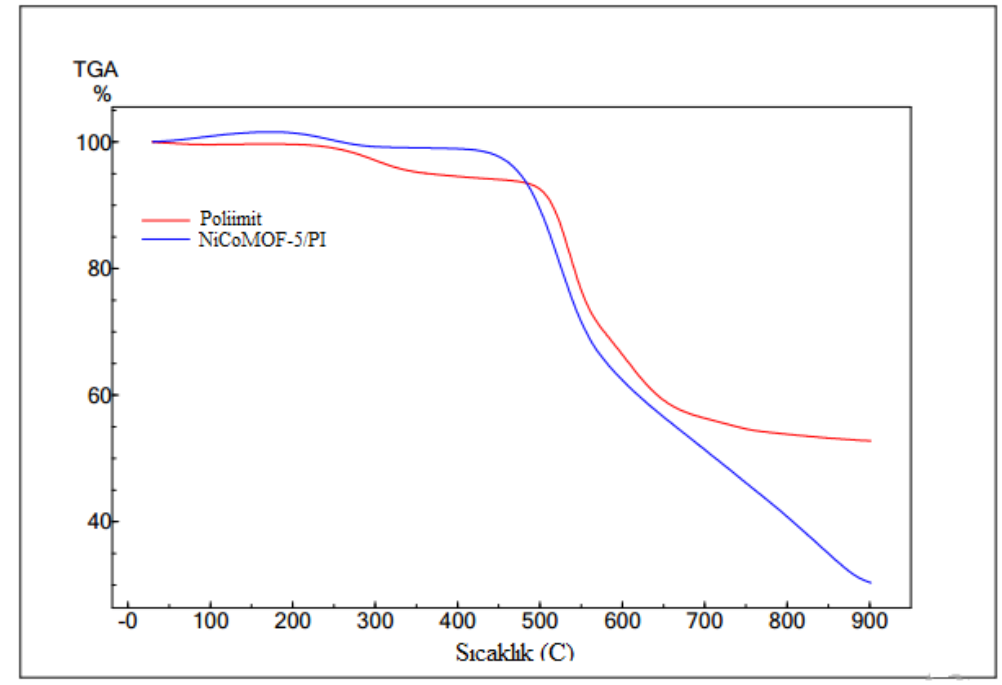

Şekil 3.2. NiCoMOF-5/PI membranın TGA eğrisi

Hazırlanan örneklerinin karakteristik yapılarını anlayabilmek için MOF-5 yapısı 650-4000 $\mathrm{cm}^{-1}$ dalga boyu aralığında toplanarak IR spektrası elde edilmiştir ve Şekil 3.3'te NiCoMOF-5'in karakteristik pikleri gösterilmiştir. 


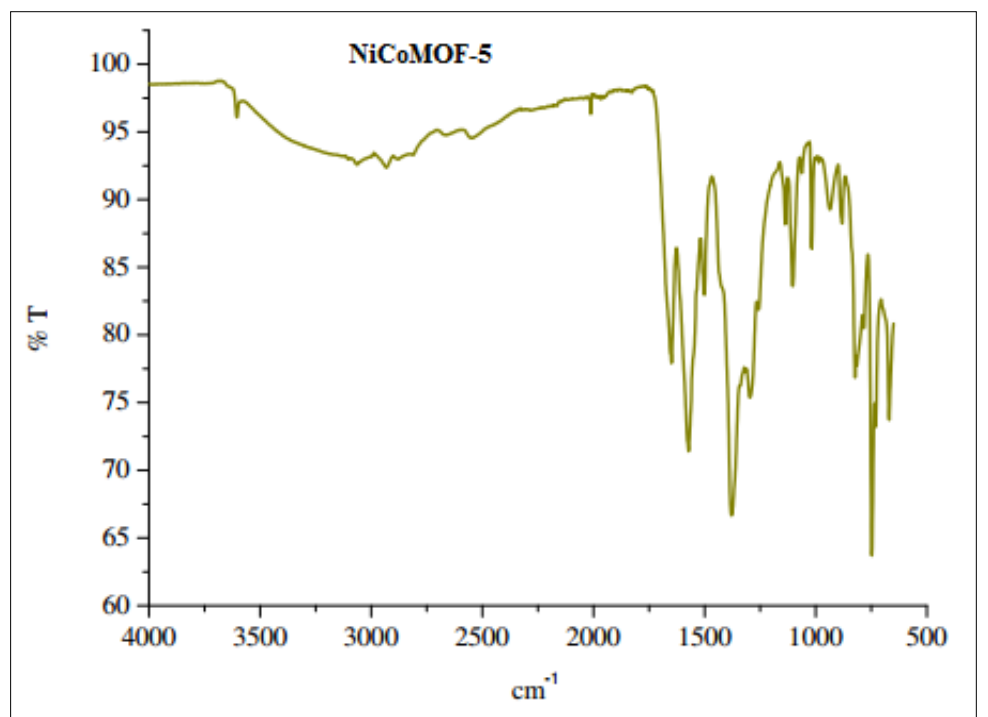

Şekil 3.3. NiCoMOF-5 yapısının IR eğrisi

Bu yapı 1400-1700 $\mathrm{cm}^{-1}$ bölgesinde karboksil fonksiyonel grubu (COO) ifade eder. $1580-1500 \mathrm{~cm}^{-1}$ aralığında karboksilik simetrik gerilme titreşimi ve $1400-1300 \mathrm{~cm}^{-1}$ aralı̆̆ında ise karboksilik asimetrik gerilme titreşimi gösterir [13 ve14]. $700-1200 \mathrm{~cm}^{-1}$ aralığındaki titreşim bandı ise terefitalat bileşiklerinin izi olarak ifade edilir [14]. Bu yapıda yer alan ve 1250 ile $1000 \mathrm{~cm}^{-1}$ dalga boyunda meydan gelen küçük pikler BDC bağlayıcısının benzen halkasında yer alan C-H grubuna karşılık gelir [6]. 2800- $3600 \mathrm{~cm}^{-1}$ aralı̆̆ındaki geniş bant ise metal koordinasyon alanındaki suyun varlığını gösterir [13 ve 14].

MOF-5 yapılarının morfolojilerini incelemek için taramalı elektron mikroskobu kullanılmıştır. MOF-5 yapısı litetatürde de belirtildiği gibi iyi tanımlanmış kübik yapılar olarak bilinmektedir [11]. Bu çalışmada amaç, MOF-5 yapısına metal bağlanmasıyla yapının değişip değişmediğini görmektir. MOF-5 ve metal katkılı MOF-5 yapılarının farklı noktalardan farklı büyütmelerle elde edilen SEM görüntüleri Şekil 3.4'te verilmiştir. Şekilden de anlaşılacağı gibi metal katkılı MOF-5 yapılarının yalın haldeki MOF-5 yapısı ile oldukça benzer bir morfoloji gösterdiğini ve metal ilavesinin MOF-5 yapısının morfolojisini değiştirmediğini ispatlamaktadır.
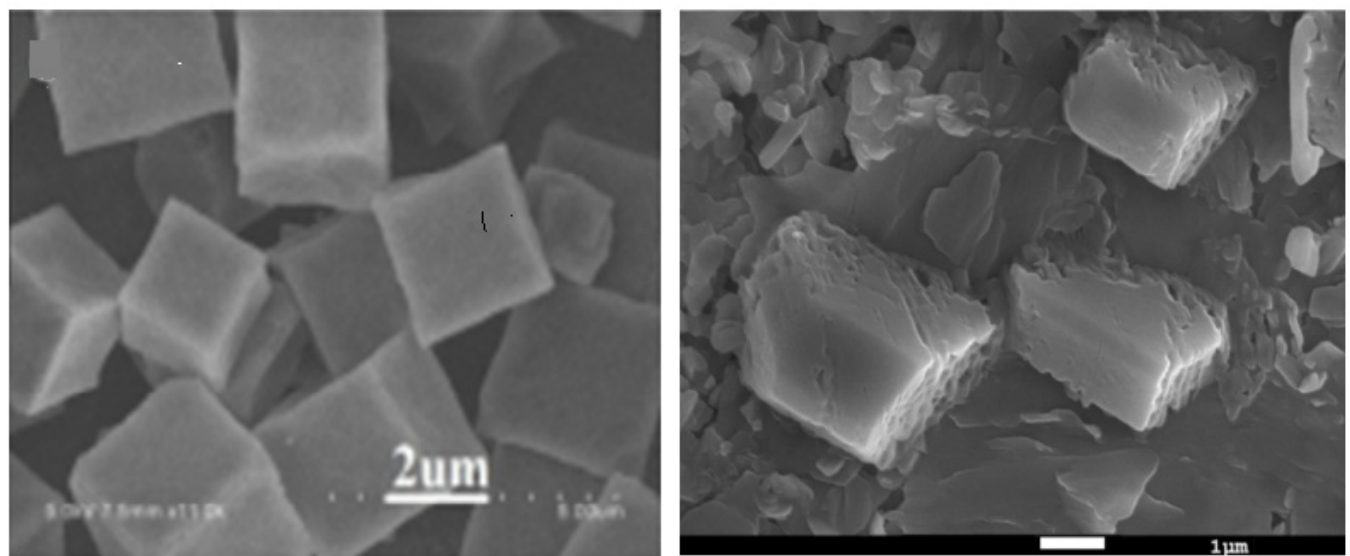

Şekil 3.4. MOF-5 ve NiCoMOF-5'in SEM görüntüsï

\subsection{Membran Gaz Geçirgenlik Deneyleri}

Saf polimer ve NiCoMOF-5 içeren membranlarla, gaz geçirgenlik deneyleri sabit hacim ve değişken basınç yöntemi uygulanarak gerçekleştirildi. Tüm ölçümler oda sıcaklığında ve 100-500 kPa basınç aralığında gerçekleştirildi. Membran düzeneğinin üst kısmından besleme yapılarak basınç dönüştürücü yardımıyla alta geçen gazın zamana karşılık basınç değişimi ölçüldü ve toplanan veriler dijital olarak kaydedildi.

Geçirgenlik değerleri aşağıdaki eşitlik [15] kullanılarak hesaplandi. 


$$
\mathrm{P}=\left(\frac{d p}{d t}\right) \frac{V T_{0}}{A \Delta P} \times \frac{L}{T P_{0}}
$$

$\mathrm{P}=$ Permeabilite, barrer $\left(1\right.$ barrer $\left.=1 \times 10^{-10} \mathrm{~cm}^{3} \mathrm{~cm} / \mathrm{cm}^{2} \mathrm{~s} \mathrm{cmHg}\right)$

$\mathrm{dp} / \mathrm{dt}=$ Geçirgenlik deneyinde elde edilen doğrunun eğimi

$\mathrm{V} \quad=$ Althacim, $\mathrm{cm}^{3}$

$\Delta \mathrm{P} \quad=$ Membran yüzeyleri arasındaki basınç farkı, $\mathrm{cmHg}$

A $\quad=$ Membran alanı, $\mathrm{cm}^{2}$

$\mathrm{L} \quad=$ Membran kalınlı $\breve{\mathrm{g}}, \mathrm{cm}$

$\mathrm{T} \quad=$ Oda sicaklığı, $\mathrm{K}$

$\mathrm{T}_{0} \quad=$ Standart sicaklık, $\mathrm{K}$

$\mathrm{P}_{0} \quad=$ Standart basınç, $\mathrm{cmHg}$

Saf polimerik membranın ve polimer yapısına \%5 yükleme oranında NiCoMOF-5 katılmasıyla oluşturulan karışık matriksli membranların gaz geçirgenlik ölçümleri oda sıcaklığında ve 5 farklı besleme basıncında gerçekleştirilmiş ve elde edilen geçirgenlik sonuçları Şekil 3.5'te verilmiştir.

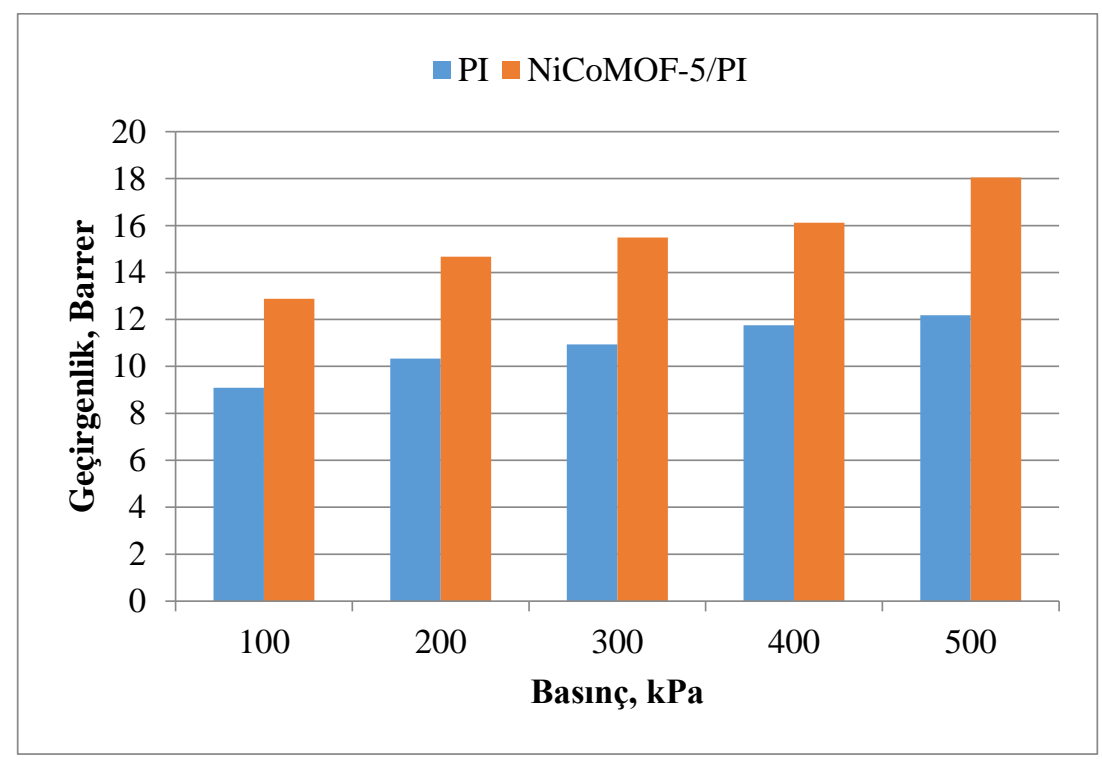

Şekil 3.5 Ağırlıkça \%5 NiCoMOF-5 içeren PI membranın farklı basınçlarda $\mathrm{H}_{2}$ geçigenliği

Basınç artışı ile birlikte hem PI hem de NiCoMOF-5/PI karışık matriksli membranında $\mathrm{H}_{2}$ geçirgenliğinde artış görülmüştür. Saf poliimit membranda $\mathrm{H}_{2}$ geçigenliği $100 \mathrm{kPa}$ 'da 9,087 barrer, $200 \mathrm{kPa}$ 'da 10,332, $300 \mathrm{kPa}$ 'da 10,935, $400 \mathrm{kPa}$ 'da 11,754 ve $500 \mathrm{kPa}$ 'da 12,177 barrer olarak bulunmuştur. Saf poliimitin $\mathrm{H}_{2}$ geçirgenliği $100 \mathrm{kPa}$ 'dan 500 kPa'a çıkılmasıyla \%34 artmıştır.

NiCoMOF-5/PI karışık matriksli membranında $\mathrm{H}_{2}$ geçigenliği $100 \mathrm{kPa}$ 'da 12,877 barrer, $200 \mathrm{kPa}$ 'da 14,673, $300 \mathrm{kPa}$ 'da 15,495, $400 \mathrm{kPa}$ 'da 16,117 ve $500 \mathrm{kPa}$ 'da 18,047 barrer olarak hesaplanmıştır. NiCoMOF-/PI membranın $\mathrm{H}_{2}$ geçirgenliği $100 \mathrm{kPa}$ 'dan $500 \mathrm{kPa}$ a çıkılmasıyla \%40 artmıştır. Sonuçlar göstermektedir ki, basınç artışıly birlikte $\mathrm{H}_{2}$ geçirgenliği NiCoMOF-5/PI membranda saf poliimit membrana göre daha yükselmiştir.

Saf polimimit ve karışık matriksli membran karşılaştıııldı̆̆ında, $100 \mathrm{kPa}$ basıncında NiCoMOF-5/PI membranın $\mathrm{H}_{2}$ geçirgenliği PI membrana göre \%41,7, $200 \mathrm{kPa}$ basıncında, \%61,47, $300 \mathrm{kPa}$ basıncında \%70,52, 400 $\mathrm{kPa}$ basıncında \%77,36, $500 \mathrm{kPa}$ basıncında \%98,60 artmıştır. $\mathrm{H}_{2}$ geçirgenlik değerinin karışık matriksli membranda yüksek olmasının nedeni, metal ilave edilmiş MOF-5' in gözenekli yapısı sayesinde gaz taşınımını kolaylaştırmasıdır.

\section{Tartışma ve Sonuç}

Bu çalışmada, solvotermal yöntemi kullanılarak NiCoMOF-5 yapısı sentezlemiş ve saf polimiit membrana ilave edilerek karışık matriksli membran üretilmiştir. Elde edilen yapıları karakterize etmek için taramalı elektron mikroskobu, fourier dönüşümlü kızılötesi spektrofotometresi ve termogravimetrik analizler yapılmıştır. İyi tanımlanmış kübik yapılar elde edilen NiCoMOF-5' in başarılı bir şekilde sentezlendiği ve literatür ile uyumlu olduğu görülmüştür. 
Ayrıca poliimite MOF ilavesinin poliimidin termal kararlılığını bozmadığı da anlaşılmıştır. Karışık matriksli membranın gaz geçirgenlik deneylerinde, PI'e MOF ilave edilmesi $\mathrm{H}_{2}$ geçirgenliğini saf poliimide göre arttırmıştır. \%5 yükleme oranında ve $500 \mathrm{kPa}$ basıncında NiCoMOF-5/PI membranın $\mathrm{H}_{2}$ geçirgenliği saf poliimidin $\mathrm{H}_{2}$ geçirgenliğinden 2.8 kat fazla olmuştur. Bu sonuç MOF yapılarının membranların $\mathrm{H}_{2}$ geçirgenliğinde büyük bir rol oynadığı ve taşınım mekanizmasına pozitif etki yarattığı söylenebilir.

\section{Kaynaklar}

[1] Dincer I., "Technical, environmental and exergetic aspects of hydrogen energy systems" International Journal of Hydrogen Energy, 27, 265-285. 2002

[2] Jain I. P., "Hydrogen the fuel for 21st century" International Journal of Hydrogen Energy, 34, 7368-7378, 2009

[3] Züttel A., "Materials for hydrogen storage" Materials Today, 6, 24-33, 2003

[4] Berg A. W. C., Arean C. O., "Materials for hydrogen storage: current research trends and perspectives" Chemical Communications, 6, 668-681, 2008

[5] Adhikari S., Fernando S.,"Hydrogen Membrane Separation Techniques" Industrial \& Engineering Chemistry Research, 45, 875-881, 2006

[6] Arjmandi M., Pakizeh M., "Mixed matrix membranes incorporated with cubic-MOF-5 for improved polyetherimide gas separation membranes: Theory and experiment" Journal of Industrial and Engineering Chemistry, 20, 3857-3868, 2014

[7] Perez E. V., Balkus J., Ferraris J. P., Musselman I. H., "Mixed-matrix membranes containing MOF-5 for gas separations" Journal of Membrane Science, 328, 165-173, 2009

[8] Li J., Cheng S., Zhao Q., Long P., Dong J., "Synthesis and hydrogen-storage behavior of metal-organic framework MOF-5" International Journal of Hydrogen Energy, 34, 1377-1382, 2009

[9] Li H., Shi W., Zhao K., Li H., Bing Y., Cheng P., "Enhanced Hydrostability in Ni-Doped MOF-5" Inorganic Chemistry, 51, 9200-9207, 2012

[10] Yang J.M., Liu Q., Sun W. Y., “Co(II)-doped MOF-5 nano/microcrystals: Solvatochromic behaviour, sensing solvent molecules and gas sorption property” Journal of Solid State Chemistry, 218, 50-55, 2014

[11] Yang J.M., Liu Q., Sun W. Y., "Shape and size control and gas adsorption of Ni(II)-doped MOF-5 nano/microcrystal” Microporous and Mesoporous Materials, 190. 26-31. 2014

[12] Ozturk B., Demirciyeva F., "Comparison of biogas upgrading performances of different mixed matrix membranes” Chemical Engineering Journal, 222, 209-217, 2013

[13] Huang, L., Wang H., Chen J., Wang Z., Sun J., Zhao D., Yan Y., "Synthesis, morphology control, and properties of porous metal-organic coordination polymers" Microporous and Mesoporous Materials, 58, 105-114, 2003

[14] Sabouni R., Kazemian H., Rohani S., "A novel combined manufacturing technique for rapid production of IRMOF-1 using ultrasound and microwave energies” Chemical Engineering Journal, 165, 966-973, 2010

[15] Weng T. H., Tseng H. H., Wey M. Y., "Fabrication and characterization of poly(phenylene oxide)/SBA15/carbon molecule sieve multilayer mixed matrix membrane for gas separation” International Journal of Hydrogen Energy, 35, 6971-6983, 2010 


\section{Extended Abstract}

\section{Introduction}

Hydrogen is one of the most important energy carrier of the future becuse of abundance in nature and its high energy density also environmental friendly properties. Since hydrogen is not pure in nature, it can be separated from the gas mixture by various techniques. Membrane technology involved in hydrogen separation processes has attracted attention in recent years thanks to its energy efficiency and mechanical strength. Membranes are classified as polymeric, inorganic and mixed matrix membranes in gas separation. However, polimeric membrane have invers relationship between permeability and selectvitiy. Inorganic membranes, on the other hand, are difficult to produce because of their expensive manufacturing, limited number of structures and they are very fragile. To solve this problem, mixed matrix membranes (MMM) emerged as an alternative to polymeric and inorganic membranes, are formed by the addition of filler into polymeric membrane. Among the fillers, metal organic framework (MOF-5) have attracted attention with their high-surface areas, numerous pore size and structure compared to other fillers. However MOF-5 is very sensitive to moisture and lead to differences in experimental results, and recent studies have shown that the addition of metal to the MOF-5 structure solves this problem and also improves the performance of the membranes. NiCoMOF-5 structure was formed with Cobalt (Co) and Nickel (Ni) metals to understand the gas permeability effect of the metals added to MOF-5 structure and this structure was added into polyimide membrane to form mixed matrix membrane. The prepared MOF structure was characterized by scanning electron microscopy (SEM), fourier transform infrared spectroscopy (FTIR) and thermogravimetric analysis (TGA).

\section{Method}

NiCoMOF-5 synthesis, mixed matrix membrane production, MOF and membrane characterization and permeability tests were performed. NiCoMOF-5 was synthesized by solvotermal method. $\mathrm{Zn}(\mathrm{NO} 3) 2 \cdot 6 \mathrm{H} 2 \mathrm{O}, \mathrm{Ni}\left(\mathrm{NO}_{3}\right)_{2} \cdot 6 \mathrm{H}_{2} \mathrm{O}$ ve $\mathrm{Co}\left(\mathrm{NO}_{3}\right)_{2} \cdot 6 \mathrm{H}_{2} \mathrm{O}$ and $\mathrm{H}_{2} \mathrm{BDC}$ were dissolved in DMF under strong agitation. After that, sample placed in an oven at $105{ }^{\circ} \mathrm{C}$ for $24 \mathrm{~h}$. NiCoMOF-5 obtained by filtering the solution. The sampple was dried for $1 \mathrm{~d}$ at $60^{\circ} \mathrm{C}$ in a vacuum oven. To fabricate pure PI membrane, PI was dissolved in NMP and stirred $\left(12 \mathrm{~h}\right.$ in $\left.70^{\circ} \mathrm{C}\right)$. Then films were casted on a clean glass plate using a casting rod. The glass plate was placed in a vacuum oven at $70^{\circ} \mathrm{C}$ and low pressure for $12 \mathrm{~h}$ to allow the solvent to evaporate completely. Then, the membrane was annealed in a vacumm oven at $220^{\circ} \mathrm{C}$ for $1 \mathrm{~d}$. The fabrication method for the MMMs was similar to the pure polymer membrane preparation with the additional step of compositing NiCoMOF-5. PI was dissolved in NMP to obtain a $10 \mathrm{wt}$ \% polymer solution. MOF-5 (loading rate is 5\%) was added to NMP. The two solutions were bath sonicated and stirred for $3 \mathrm{~h}$ then were mixed by pouring the polymer solution into the NiCoMOF-5 solution. The combined solution was stirred and bath sonicated for 5 h. Other steps for the fabrication of MMMs were identical to that of the pure polymer membrane preparation.

\section{Results and Discussion}

In this study, thermal analysis techniques were applied to determine the thermal stability of MOF-5. The thermal stability of the mixed matrix membrane exhibits very similar curves to pure polyimide. This result shows that the thermal stability of the structure does not change when NiCoMOF-5 is added to the polyimide structure. In order to understand the characteristics structure of the prepared sample, IR spectra were obtained. Scanning electron microscopy was used to study the morphology of NiCoMOF-5 structure. Metal addition into MOF-5 structure did not alter morphology and similar morphology was observed. Gas permeability measurements of pure polymeric membrane and mixed matrix membrane were performed at room temperature and at 5 different feed pressures. $\mathrm{H}_{2}$ permeabilities in both PI and NiCoMOF-5/PI mixed matrix membrane have increased with increasing pressure. In gas permeability experiments of mixed matrix membrane, the addition of MOF into PI increased $\mathrm{H}_{2}$ permeability compared to pure polyimide. At a loading rate of $5 \%$ and a pressure of $500 \mathrm{kPa}$, the $\mathrm{H}_{2}$ permeability of the NiCoMOF-5/PI membrane was 2,8 times greater than the permeability of the pure polyimide. This result suggests that MOF structures play a major role in the $\mathrm{H}_{2}$ permeability of the membranes and have a positive effect on the transport mechanism. 